\title{
Effects of Different Practice Task Constraints on Fluctuations of Player Heart Rate in Small-Sided Football Games
}

\author{
R. Duarte ${ }^{1,2, *}$, D. Araújo ${ }^{2}$, O. Fernandes ${ }^{1}$, B. Travassos ${ }^{2,3}$, H. Folgado ${ }^{1}$, A. Diniz ${ }^{2}$ and K. Davids ${ }^{4}$ \\ ${ }^{I}$ Department of Sport and Health, University of Évora, Portugal \\ ${ }^{2}$ Faculty of Human Kinetics, Technical University of Lisbon, Portugal \\ ${ }^{3}$ University of Beira Interior, Portugal \\ ${ }^{4}$ School of Human Movement Studies, Queensland University of Technology, Australia
}

\begin{abstract}
This paper analyzes effects of different practice task constraints on heart rate (HR) variability during 4v4 smallsided football games. Participants were sixteen football players divided into two age groups (U13, Mean age: $12.4 \pm 0.5$ yrs; U15: 14.6 \pm 0.5$)$. The task consisted of a $4 \mathrm{v} 4$ sub-phase without goalkeepers, on a $25 \times 15$ m field, of 15 minutes duration with an active recovery period of 6 minutes between each condition. We recorded players' heart rates using heart rate monitors (Polar Team System, Polar Electro, Kempele, Finland) as scoring mode was manipulated (line goal: scoring by dribbling past an extended line; double goal: scoring in either of two lateral goals; and central goal: scoring only in one goal). Subsequently, \%HR reserve was calculated with the Karvonen formula. We performed a time-series analysis of HR for each individual in each condition. Mean data for intra-participant variability showed that autocorrelation function was associated with more short-range dependence processes in the "line goal" condition, compared to other conditions, demonstrating that the "line goal" constraint induced more randomness in HR response. Relative to inter-individual variability, line goal constraints demonstrated lower \%CV and \%RMSD (U13: 9\% and 19\%; U15: 10\% and 19\%) compared with double goal (U13: $12 \%$ and 21\%; U15: $12 \%$ and 21\%) and central goal (U13: $14 \%$ and 24\%; U15: $13 \%$ and $24 \%$ ) task constraints, respectively. Results suggested that line goal constraints imposed more randomness on cardiovascular stimulation of each individual and lower inter-individual variability than double goal and central goal constraints.
\end{abstract}

Keywords: Task constraints; time-series analysis; fluctuations.

\section{INTRODUCTION}

Association football is a team sport that incorporates frequent fluctuations between high and low exercise intensities [1]. Heart rate (HR) responses have been used to grade the exercise intensity in a range of sports, and also for evaluating training activities in football [2]. More recently, heart rate has been used to discriminate differences between training regimes. For example, it has been used to evaluate the effectiveness of generic (interval running) versus specific (smallsided games) training modes in physiological and performance indicators [3], or to compare the physiological response to several types of small-sided games constrained by differences in coach encouragement, number of players involved and/or field dimensions [4].

These comparisons may, however, be criticized on methodological grounds because of the inherent variability of physiological responses to match-play and small-sided games [1,5]. Obtaining approximate mean and standard deviations of physiological measures in small-sided games for comparative purposes may not constitute evidence that the physiological demands of two exercise stressors are similar

*Address correspondence to this author at the Department of Sport and Health, University of Évora, Portugal; Tel: 00351214196777;

Fax: 00351214144712; E-mail: rduarte@ fmh.utl.pt
$[1,6]$. Cardiovascular demands on the players contain highly variable and unpredictable fluctuations that can be measured as a result of non-linear interactions between a performer and environmental constraints [7]. From an ecological dynamics perspective, task constraints are a particular set of imposed environment conditions that can be used to manipulate the relationship between performer-environment interactions towards an intended outcome [7]. Based on this approach, the aim of this paper was to analyze the effects of different practice task constraints on player heart rate variability during $4 \mathrm{v} 4$ small-sided football games.

\section{METHODS}

Sixteen football players divided into two age groups (U13, Mean age: $12.4 \pm 0.5$ yrs; U15: $14.6 \pm 0.5$ yrs) performed in a small-sided game under three different practice task constraints. Each group completed the protocol during regular training sessions, 96 hours after their last game. The task consisted of a $4 \mathrm{v} 4$ sub-phase without goalkeepers, on a $25 \times 15 \mathrm{~m}$ field, of 15 minutes duration with an active recovery period of 6 minutes between each condition (see Fig. 1). The $4 \mathrm{v} 4$ version of football was chosen because of evidence on its efficacy as a practice game mode for fitness conditioning [8]. This protocol was preceded by a patterned warm-up of 10 minutes. We recorded players' heart rates using heart rate monitors at $0.2 \mathrm{~Hz}$ (Polar Team System, Polar Electro, Kem- 
pele, Finland). The task constraint of scoring mode was manipulated in three conditions: i) line goal, scoring by dribbling past an extended line; ii) double goal, scoring in either of two lateral goals; and iii), central goal, scoring only in one goal. To ensure that there were minimal stoppages in play during all drill conditions, 10 footballs were maintained around the playing area to encourage quick restarts by the players. Subsequently, \%HR reserve was calculated with the Karvonen formula. Preliminary analysis showed that HR during the break after the initial warm-up was similar to the other two rest periods $(\mathrm{P} \geq 0.05)$. After confirmation of mean differences between active breaks and exercise bouts $(\mathrm{P}<0.05)$, rest periods were excluded from analysis. We next performed a time-series analysis of HR for each individual in each condition. To analyze intra-participant variability we used an autocorrelation technique [9]. To examine interparticipant variability we used percentage of coefficient of variation $(\% \mathrm{CV})$ and percentage of root mean square difference (\%RMSD) [10].

\section{RESULTS}

Mean data of intra-participant variability showed that autocorrelation function was associated with more shortrange dependence processes in the "line goal" condition, compared to other conditions. Fig. (2) demonstrates for both groups that the increase in the lag resulted in a major slope associated with the line goal task constraint, followed by double goal and the central goal task constraint.

Relative to inter-individual variability, \%CV and \% RMSD exhibited lower values in the line goal task constraint, followed by double goal and central goal as showed in Table $\mathbf{1}$.

\section{DISCUSSION}

The findings of the present study suggest that coaches can change cardiovascular stimulation of youth soccer players by modifying the key task constraint of goal scoring mode in training. Results suggested that line goal constraints

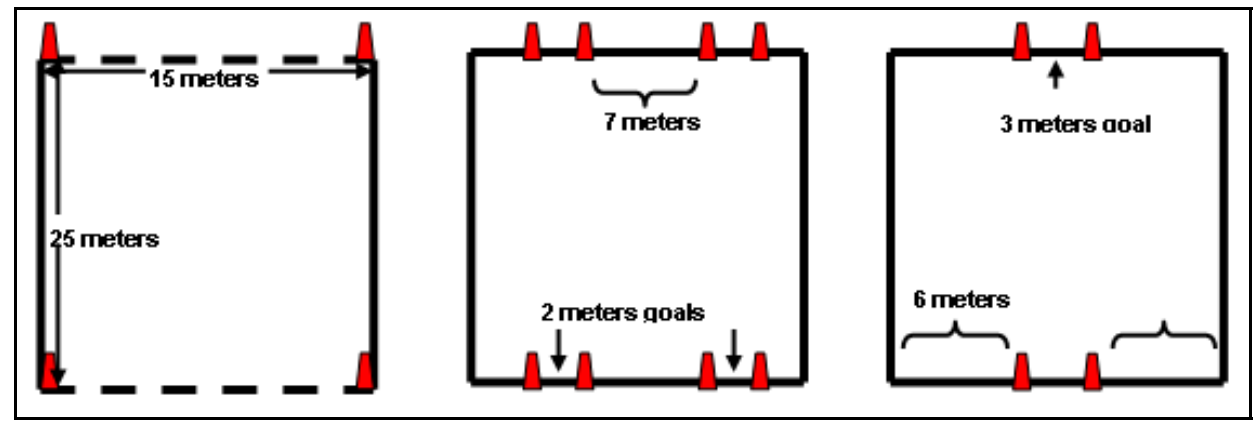

Fig. (1). Experimental task schematic representation. From the left to the right, figure shows line goal, double goal and central goal conditions, respectively.
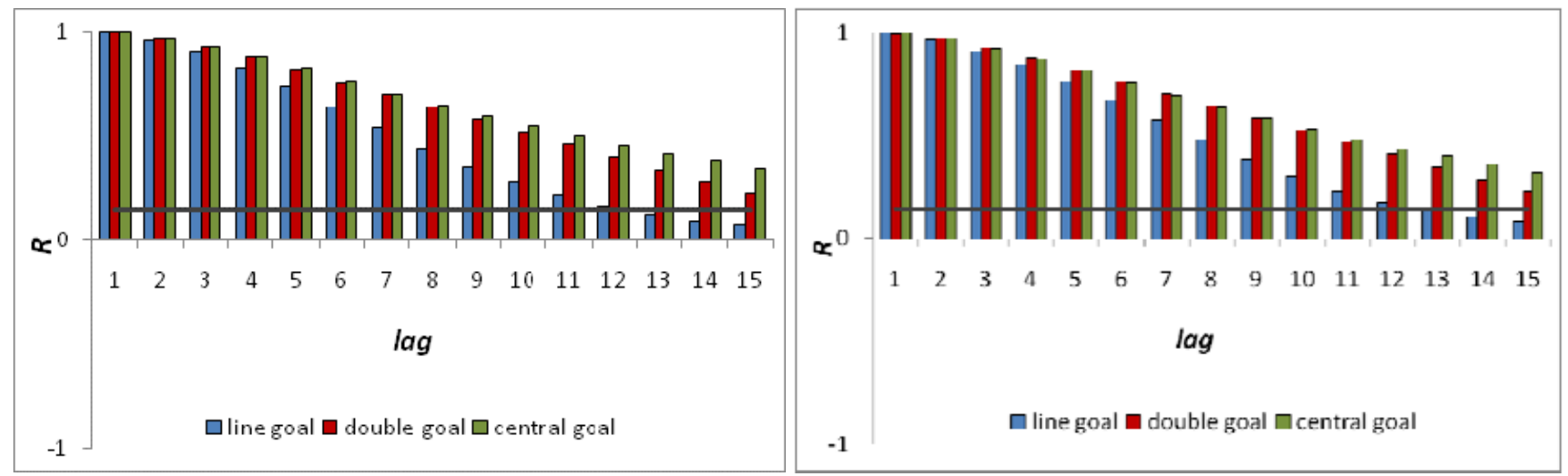

Fig. (2). Mean data from autocorrelation technique for Under 13 (left) and for Under 15 (right).

Table 1. Inter-Participant Variability in the Three Practice Task Constraints

\begin{tabular}{|c|c|c|c|}
\hline & Line Goal & Double Goal & Central Goal \\
\hline U13 & $\begin{array}{c}\text { CV: } 9 \% \\
\text { RMSD: } 19 \%\end{array}$ & $\begin{array}{c}\text { CV: } 12 \% \\
\text { RMSD: } 21 \%\end{array}$ & $\begin{array}{c}\text { CV: } 14 \% \\
\text { RMSD: } 24 \%\end{array}$ \\
\hline U15 & $\begin{array}{l}\text { CV: } 10 \% \\
\text { RMSD: } 19 \%\end{array}$ & $\begin{array}{c}\text { CV: } 12 \% \\
\text { RMSD: } 21 \%\end{array}$ & $\begin{array}{c}\text { CV: } 13 \% \\
\text { RMSD: } 24 \%\end{array}$ \\
\hline
\end{tabular}


imposed more randomness in cardiovascular stimulation of all individuals. This type of stimulation reproduces more accurately the fluctuations and the intermittent profile of top players' activity in football [5]. Thus, this constraint can stimulate the development of individual skills in 1-on-1 and 1-on-many sub-phases with a high level of unpredictability in the cardiovascular system stimulation. By introducing the double goal constraint in small-sided football games, coaches can also increase the variability in physiological stimulation of the players relative to more traditional central goal drills. This strategy can assemble the beneficial effects of developing support and game vision with a remarkable variability in physiological stimulation.

Results also suggested that inter-individual variability associated with the line goal task constraint is lower than that associated with both double goal and central goal constraints. These effects represent more standardized cardiovascular stimulation of the players involved in the line goal task. By using the line goal task constraint, youth coaches can also guarantee more similar physiological stimulation of all players involved in training. The equivalent cardiovascular response of U13 and U15 suggests that the practice drills imposed similar variability in physiological stimulation, independent of the chronological age of the players.

Quantifying neurobiological variability in human movement can lead us to understand how different practice task constraints affect the dynamics of performer-environment interactions in sport [11]. The results of the present study suggest that the manipulation of the key task constraint of scoring mode in small-sided football games can be a useful strategy for youth soccer coaches to set the required variability in exercise intensity, while providing tools for enhancing tactical aspects of the game in team games players.

\section{REFERENCES}

[1] Drust B, Atkinson G, Reilly T. Future perspectives in the evaluation of the physiological demands of soccer. Sports Med 2007; 37: 783-805.

[2] Esposito F, Impellizzeri FM, Margonato V, Vanni R, Pizzini G, Veicsteinas A. Validity of heart rate as an indicator of aerobic demand during soccer activities in amateur soccer players. Eur J Appl Physiol 2004; 93: 167-72.

[3] Impellizzeri FM, Marcora SM, Castagna C, et al. Physiological and performance effects of generic versus specific aerobic training in soccer players. Int J Sports Med 2006; 27: 483-92.

[4] Rampinini E, Impellizzeri FM, Castagna C, et al. Factors influencing physiological responses to small-sided soccer games. J Sports Sci 2007; 25: 659-66.

[5] Bangsbo J. The physiology of soccer with special reference to intense intermittent exercise. Acta Physiol Scand 1994; 15 (Suppl. 159): 1-155.

[6] Duarte R, Batalha N, Folgado H, Sampaio J. Effects of exercise duration and number of players in heart rate responses and technical skills during futsal small-sided games. Open Sports Sci 2009; 2: 37-1.

[7] Araújo D, Davids K, Hristovski R. The ecological dynamics of decision making in sport. Psychol Sport Exerc 2006; 7: 65376.

[8] Jones S, Drust B. Physiological and technical demands of $4 \mathrm{v} 4$ and 8v8 games in elite youth soccer players. Kinesiology 2007; 39: 150-6.

[9] Wagenmakers E, Farrell S, Ratcliff R. Estimation and interpretation of 1/fa noise in human cognition. Psychol Bull Rev 2004; 11: $579-15$.

[10] Mullineaux D, Bartlett R, Bennett S. Research design and statistics in biomechanics and motor control. J Sports Sci 2001; 19: 739-60.

[11] Davids K, Button C, Bennett S. Dynamics of skill acquisition: a constraints-led approach. Champaign: Human Kinetics 2008.

Received: July 05, 2009

Revised: October 10, 2009

Accepted: December 05, 2009

() Duarte et al.; Licensee Bentham Open.

This is an open access article licensed under the terms of the Creative Commons Attribution Non-Commercial License

(http://creativecommons.org/licenses/by-nc/3.0/) which permits unrestricted, non-commercial use, distribution and reproduction in any medium, provided the work is properly cited. 